\title{
Growth, Habit Formation, and Catching-up with the Joneses*
}

\author{
Jaime Alonso-Carrera \\ Departamento de Economia Aplicada \\ Universidade de Vigo \\ Jordi Caballé \\ Unitat de Fonaments de l'Anàlisi Economica and CODE \\ Universitat Autònoma de Barcelona \\ Xavier Raurich \\ Departament d'Economia \\ Universitat de Girona
}

Revised version: June 16, 2003

\begin{abstract}
When habits are introduced multiplicatively in a capital accumulation model, the consumers' objective function might fail to be concave. In this paper we provide conditions aimed at guaranteeing the existence of interior solutions to the consumers' problem. We also characterize the equilibrium path of two growth models with multiplicative habits: the internal habit formation model, where individual habits coincide with own past consumption, and the external habit formation (or catchingup with the Joneses) model, where habits arise from the average past consumption in the economy. We show that the introduction of external habits makes the equilibrium path inefficient during the transition towards the balanced growth path. We characterize in this context the optimal tax policy.
\end{abstract}

Key words: endogenous growth, habit formation, consumption externalities, optimal taxation.

JEL Classification Number: E21, O40.

${ }^{*}$ We thank the anonymous referees of this review and the seminar participants at CEMFI for their valuable comments. Of course, they should not bear any responsibility for the remaining errors. Financial support from the Spanish Ministry of Science and Technology through grants SEC2000-0684, BEC200201995 and HI2001-0039, the Generalitat of Catalonia through the Barcelona Economics program (CREA) and grant SGR2001-00162 is gratefully acknowledged.

Correspondence address: Jordi Caballé. Universitat Autònoma de Barcelona. Departament d'Economia i d'Història Econòmica. Edifici B. 08193 Bellaterra (Barcelona). Spain.

Phone: (34)-935.812.367. Fax: (34)-935.812.012. E-mail: Jordi.Caballe@uab.es 


\section{Introduction}

Several recent papers have introduced habit formation in the agents' utility function in order to explain some empirical facts that cannot be reconciled with the traditional models displaying time-separable preferences. Examples of this strand of the literature are the papers of Abel (1990, 1999), who provides a possible explanation of the equity premium puzzle; Lettau and Uhlig (2000), who try to fit some stylized facts of business cycles; Ljungqvist and Uhlig (2000), who examine the effects of fiscal policy under habit formation; Fuhrer (2000), who studies the implications of habit formation for monetary policy; and Carroll et al. (1997, 2000) and Shieh et al. (2000), who study how the patterns of growth are modified when habits are present.

The aim of the present paper is twofold. First, to characterize the equilibrium path of a class of endogenous growth models under habit formation and, second, to characterize the optimal tax rates that solve the inefficiencies brought about by the habits associated with the average past consumption of the economy. In order to allow for sustained growth, we will assume that the production function is asymptotically linear in capital in spite of potentially exhibiting diminishing returns with respect to capital (as in Jones and Manuelli, 1990). This type of "Sobelow" production function constitutes one of the main differences of our analysis with the related literature. On the one hand, Carroll et al. $(1997,2000)$ characterize the equilibrium path when the production function is linear in capital and, hence, transitional dynamics is driven only by habits. In contrast, in our paper, transitional dynamics is driven by both habit formation and diminishing returns to scale. We show that this difference modifies the patterns of growth along the transition. On the other hand, Fisher and Hof (2000) analyze the optimal tax policy in a model without habit formation where agents are exposed to contemporaneous consumption spillovers. Therefore, unlike in our model, transitional dynamics is driven only by the neoclassical production function they consider, which in turn prevents the economy from exhibiting sustained growth.

In our model we will assume that consumers' utility depends both on own current consumption and a reference level. This reference is a standard of living determined by the own past consumption and by the past average consumption of the economy. While the isoelastic functional form for the individuals' instantaneous utility has been extensively used in the literature, two alternative forms have been used to introduce habits. One form is the "additive" one, according to which habits play in fact the role of a minimum level of consumption. The other functional form is the "multiplicative" one, where consumers' utility depends on their current level of consumption relative to a reference level determined by habits. Both functional forms exhibit some technical problems. On the one hand, Carroll (2000) points out that the additive model may give rise to a not well defined utility in stochastic economies under plausible calibrations. On the other hand, we argue in this paper that the instantaneous utility function is not concave under our multiplicative formulation for internal habits. In this case, the convexity of the consumers' maximization problem is not ensured and the optimal path chosen by consumers might fail to be interior. However, we provide a set of assumptions under which the standard first order conditions characterize interior equilibrium paths. We also show that those assumptions are met by two famous models, which are in 
fact extreme cases of our original model: the internal habit formation model, where the reference variable coincides with own past consumption, and the external habit formation (or "catching-up with the Joneses") model, where the reference is an externality accruing from the average past consumption in the economy. ${ }^{1}$

We also characterize the equilibrium path of the previous two extreme models and show that in both cases the dynamic equilibrium converges asymptotically to a balanced growth path, along which output, consumption and capital grow at a common constant rate. We show that the introduction of habits increases this long run rate of growth because it raises the consumers' willingness to shift consumption from the present to the future. We also prove that the growth rates of both models exhibit a monotonic behavior along the transition when the production function is linear in capital, whereas they could exhibit a non-monotonic behavior when the production function has diminishing returns to scale with respect to capital. In the latter case, we show that the transitional dynamics depends on the values of both capital and the reference level of consumption, whereas the transition only depends on the ratio of capital to the reference level of consumption when the production function has constant returns to scale. This means that the specification of the production function has interesting implications on cross-country convergence.

Our analysis shows that the introduction of a consumption externality makes the equilibrium of the catching-up with the Joneses model inefficient during the transition towards the balanced growth path. In order to prove this result, we use the fact that the equilibrium solution of the internal habit formation model corresponds to the efficient solution of the catching-up with the Joneses model. Then, we show that the equilibrium solutions obtained in the two models coincide in the long run, whereas these two solutions are different during the transition. Inefficiency arises because the consumers' willingness to substitute consumption across periods in the catching-up with the Joneses model is not optimal. This source of inefficiency can be corrected by means of an appropriate tax policy and, in particular, we show that either a consumption tax or an income tax may restore efficiency. If the consumers' willingness to shift present consumption to the future is suboptimally low, then the optimal fiscal policy will consist of either a decreasing sequence of consumption taxes or a subsidy on income (or output). A decreasing sequence of consumption taxes implies that future consumption purchases will be cheaper. Therefore, this fiscal policy increases consumers' willingness to postpone consumption. A subsidy on output also corrects the inefficiency because it encourages consumers to shift consumption from the present to the future. Conversely, either an increasing sequence of consumption taxes or a tax on income will be the optimal fiscal policies when consumers' willingness to shift present consumption to the future is suboptimally large.

Finally, we show that if the marginal productivity of capital is constant during the transition, which occurs when the production function is of the $A k$ type, then the optimal tax rates will depend only on the initial value of the ratio of capital to habits. However, if the marginal productivity of capital is changing during the transition, then the value of the optimal tax rates will depend on the particular initial values of capital and habits and not only on their ratio. In this case, the optimal path of tax rates could exhibit a non-monotonic behavior along its transition.

The characterization of the optimal income tax rate highlights the dynamic nature of the inefficiency, which affects the willingness to substitute consumption across periods

\footnotetext{
${ }^{1}$ This is in contrast to the "keeping-up with the Joneses" model, where the externality accrues from average current consumption (see Galí, 1994)
} 
and, thus, modifies the pattern of capital accumulation. In this respect, let us mention that Ljungqvist and Uhlig (2000) have also analyzed the inefficiency accruing from external habits. However, they consider a model without capital accumulation where the externality distorts the intratemporal choice between consumption and leisure and, thus, their efficiency analysis cannot be extended to a growth model like ours.

The rest of the paper is organized as follows. Section 2 describes the model and provides conditions that guarantee the existence of interior equilibria characterized by standard first order conditions. Section 3 and 4 characterize the equilibrium path of the internal habit formation model and of the catching-up with the Joneses model, respectively. Section 5 shows that the equilibrium of the latter model is inefficient and derives the optimal tax policy aimed at restoring efficiency. Section 6 concludes the paper. Some lengthy proofs appear in the Appendix.

\section{The Model}

Consider an economy in discrete time populated by identical dynasties facing an infinite horizon. The members of each dynasty are also identical. We assume that population grows at a constant exogenous rate $n>-1$. We also assume that consumers' utility in period $t$ depends both on consumption $c_{t}$ and on a variable $v_{t}$ representing a standard of living that it is used as a reference with respect which present consumption is compared to. This standard of living is determined by the past consumption experience. Following Abel (1990), we assume that

$$
v_{t}=c_{t-1}^{\theta} \bar{c}_{t-1}^{1-\theta},
$$

where $\theta \in[0,1], c_{t-1}$ is the own consumption in period $t-1$, and $\bar{c}_{t-1}$ is the average consumption of the economy in period $t-1$. When $\theta=1$ this formulation coincides with that of the internal habit formation $(\mathrm{IH})$ model, where the reference is just the own past consumption. On the contrary, the case where $\theta=0$ corresponds to the catching-up with the Joneses (CJ) model, where consumers' utility depends both on present consumption and on an externality accruing from the others' past consumption. Following Abel (1990) and Carroll et al. (1997), we introduce multiplicatively the reference variable. This means that consumers' utility depends on own current consumption relative to the standard of living summarized by the variable $v_{t}$. Accordingly, the instantaneous utility function takes the following functional form:

$$
u_{t}=\frac{1}{1-\sigma}\left(\frac{c_{t}}{v_{t}^{\gamma}}\right)^{1-\sigma}, \quad \text { with } \sigma>0 \text { and } \gamma \in(0,1)
$$

where $\gamma$ is a parameter measuring the importance of the consumption reference and $\sigma$ coincides with the inverse of the elasticity of intertemporal substitution of consumption when $\gamma=0$. The assumption $\gamma>0$ agrees with our notion of a reference for consumption, whereas we must impose that $\gamma<1$ since, otherwise, the utility function would not be strictly increasing in consumption along a balanced growth path. Note that if $\gamma=0$ the utility function $u$ is time-independent and concave. However, when $\gamma>0$ the utility function is time-dependent and it is not jointly concave with respect to the two variables $c_{t}$ and $v_{t}$. In fact, the necessary conditions for joint concavity are $1+\gamma(1-\sigma) \leq 0$ and $\gamma+\sigma(1-\gamma) \leq 0$. Obviously, the latter inequality cannot hold under our parametric assumptions. 
Each consumer is endowed with $k_{t}$ units of capital that are used to produce a certain amount of output according to the following Sobelow gross production function per capita:

$$
f\left(k_{t}\right)=A k_{t}+B k_{t}^{\beta},
$$

where $A>0, B \geq 0$ and $\beta \in(0,1)$. This production function allows for sustained growth provided the asymptotic marginal productivity $A$ of capital is sufficiently high (Jones and Manuelli, 1990). The output may be used either for consumption or for investment in new capital. Thus, the resource constraint per capita is given by

$$
f\left(k_{t}\right) \geq c_{t}+(1+n) k_{t+1}-(1-\delta) k_{t}, \quad \text { for all } t=0,1, \ldots
$$

where $\delta \in[0,1]$ is the depreciation rate of capital. ${ }^{2}$

The objective of each dynasty is to maximize the discounted sum of utilities of each of its identical members,

$$
\sum_{t=0}^{\infty}\left(\frac{1}{1+\rho}\right)^{t} u_{t}
$$

where $\rho>0$ is the subjective discount rate. At time $t=0$, each dynasty chooses $\left\{c_{t}, k_{t+1}\right\}_{t=0}^{\infty}$ to maximize (5) subject to (4), taking as given the path of average consumption $\left\{\bar{c}_{t}\right\}_{t=-1}^{\infty}$ and the two initial conditions on capital $k_{0}>0$ and past consumption $c_{-1}>0$. Both $c_{t}$ and $k_{t}$ are restricted to be non-negative in all periods. Note that in this dynamic optimization problem $k_{t}$ and $v_{t}$ are the state variables. While the former variable is only affected by the individual decisions of consumers, the latter is determined by both individual decisions and the exogenous path of average consumption.

The Lagrangian associated with the dynasty problem is

$$
L(c, k, \lambda)=\sum_{t=0}^{\infty}\left(\frac{1}{1+\rho}\right)^{t} u_{t}+\sum_{t=0}^{\infty} \lambda_{t}\left[f\left(k_{t}\right)-c_{t}-(1+n) k_{t+1}+(1-\delta) k_{t}\right]
$$

where $c=\left\{c_{t}\right\}_{t=0}^{\infty}, k=\left\{k_{t}\right\}_{t=0}^{\infty}$ and $\lambda=\left\{\lambda_{t}\right\}_{t=0}^{\infty}$ are non-negative paths, and the average consumption path $\left\{\bar{c}_{t},\right\}_{t=-1}^{\infty}$ is taken as given. Computing the derivative of the previous Lagrangian with respect to $c_{t}$, we obtain the following necessary first order conditions for optimality:

$$
\frac{\partial L}{\partial c_{t}}=\left(\frac{1}{1+\rho}\right)^{t} \frac{\partial u_{t}}{\partial c_{t}}+\left(\frac{1}{1+\rho}\right)^{t+1} \frac{\partial u_{t+1}}{\partial v_{t+1}} \frac{\partial v_{t+1}}{\partial c_{t}}-\lambda_{t} \leq 0
$$

with $c_{t} \geq 0$, and

$$
\frac{\partial L}{\partial c_{t}} \cdot c_{t}=0
$$

The corresponding transversality condition is

$$
\lim _{t \rightarrow \infty}\left\{\left(\frac{1}{1+\rho}\right)^{t} \frac{\partial u_{t}}{\partial c_{t}}-\lambda_{t}\right\} \leq 0,
$$

with

$$
\lim _{t \rightarrow \infty}\left\{\left[\left(\frac{1}{1+\rho}\right)^{t} \frac{\partial u_{t}}{\partial c_{t}}-\lambda_{t}\right] c_{t}\right\}=0 .
$$

\footnotetext{
${ }^{2}$ From now on, the expression "for all $t=0,1, \ldots$ " will be skipped as long as the meaning is clear.
} 
Differentiating (6) with respect to $k_{t+1}$, we also get the following necessary first order conditions:

$$
\frac{\partial L}{\partial k_{t+1}}=-(1+n) \lambda_{t}+\left[1+f^{\prime}\left(k_{t+1}\right)-\delta\right] \lambda_{t+1} \leq 0,
$$

with $k_{t+1} \geq 0$, and

$$
\frac{\partial L}{\partial k_{t+1}} \cdot k_{t+1}=0
$$

The corresponding transversality condition is

$$
\lim _{t \rightarrow \infty}\left\{-(1+n) \lambda_{t}\right\} \leq 0
$$

with

$$
\lim _{t \rightarrow \infty}\left\{-(1+n) \lambda_{t} k_{t+1}\right\}=0 .
$$

Finally, by taking the derivative of (6) with respect to the Lagrange multiplier $\lambda_{t}$, the solution to the optimization problem involves also to satisfy the resource constraint (4), and

$$
\lambda_{t} \cdot\left[f\left(k_{t}\right)-c_{t}-(1+n) k_{t+1}+(1-\delta) k_{t}\right]=0,
$$

with $\lambda_{t} \geq 0$.

The following lemma provides a necessary condition to be satisfied by an interior solution to this dynamic optimization problem:

Lemma 1 If the path $\left\{c_{t}, k_{t+1}\right\}_{t=0}^{\infty}$ chosen by a dynasty is strictly positive, then the following condition must be satisfied:

$$
\frac{1}{1+\rho}\left[\frac{\frac{\partial u_{t+1}}{\partial c_{t+1}}+\left(\frac{1}{1+\rho}\right)\left(\frac{\partial u_{t+2}}{\partial v_{t+2}}\right)\left(\frac{\partial v_{t+2}}{\partial c_{t+1}}\right)}{\frac{\partial u_{t}}{\partial c_{t}}+\left(\frac{1}{1+\rho}\right) \frac{\partial u_{t+1}}{\partial v_{t+1}} \frac{\partial v_{t+1}}{\partial c_{t}}}\right]=\frac{1+n}{1+f^{\prime}\left(k_{t+1}\right)-\delta} .
$$

Proof. Since, by assumption, $c_{t}>0$ and $k_{t}>0$, for all $t,(8)$ implies that (7) holds with equality, and similarly (12) implies that (11) also holds with equality. From combining the first order conditions (7) and (11), it is straightforward to obtain the equation (16).

The optimality condition (16), dubbed the Keynes-Ramsey equation, equates the marginal rate of substitution of consumption between periods $t$ and $t+1\left(M R S_{t, t+1}\right.$, henceforth) with the corresponding marginal rate of transformation $\left(M R T_{t, t+1}\right.$, henceforth). Note that the $M R S_{t, t+1}$ depends on the own consumption and on the externalities arising from average past consumption. ${ }^{3}$ More precisely, the $M R S_{t, t+1}$ is a function of $c_{t-1}, c_{t}, c_{t+1}, c_{t+2}$, and of the average consumptions $\bar{c}_{t-1}, \bar{c}_{t}$ and $\bar{c}_{t+1}$. Since the path $\left\{c_{t}, k_{t+1}\right\}_{t=0}^{\infty}$ chosen by a dynasty is a function of the average consumption path $\left\{\bar{c}_{t}\right\}_{t=-1}^{\infty}$, the next definition makes clear the fixed point nature of a competitive equilibrium:

Definition 1 An equilibrium path $\left\{c_{t}, k_{t+1}\right\}_{t=0}^{\infty}$ is a solution to the dynastic optimization problem when $\bar{c}_{t}=c_{t}$, for all $t$.

\footnotetext{
${ }^{3}$ It should be pointed out that, if $\sigma=1$, then the $M R S_{t, t+1}$ does not depend on the externalities, so that average past consumption does not modify the path chosen by the dynasty.
} 
From the previous definition, it follows that along the equilibrium path the $M R S_{t, t+1}$ depends only on $c_{t-1}, c_{t}, c_{t+1}$ and $c_{t+2}$. Note that this equilibrium $M R S_{t, t+1}$ differs from the $M R S_{t, t+1}$ appearing in standard models of capital accumulation because here consumers take into account the effect that present consumption has in setting the reference for next period consumption. Because of the dependence of the $M R S_{t, t+1}$ on consumption in different time periods, the analysis of the equilibrium is simplified by introducing the following transformed variables: $x_{t}=\frac{c_{t}}{c_{t-1}}, h_{t}=\frac{u_{t+1}}{u_{t}}, z_{t}=\frac{k_{t}}{c_{t-1}}$, and $m_{t}=\frac{f\left(k_{t}\right)}{k_{t}}$. Note that the average productivity $m_{t}$ of capital and the ratio $z_{t}$ of capital to the reference level of consumption are the state variables, whereas the gross rate $x_{t}$ of consumption growth and the gross rate $h_{t}$ of growth of the utility are the control variables. Note also that, for given values $c_{-1}$ and $k_{0}$ of initial past consumption and initial capital, respectively, there is a one-to-one correspondence between the equilibrium values of the original variables $c_{t}$ and $k_{t}$ and the values of the transformed variables $m_{t}$, $x_{t}, z_{t}$ and $h_{t}$. Thus, given the initial conditions $m_{0}=f\left(k_{0}\right) / k_{0}$ and $z_{0}=k_{0} / c_{-1}$, we can rewrite the equilibrium path in terms of the transformed variables.

We define a stationary path in terms of the previous transformed variables as follows:

Definition $2 A$ stationary path $\left\{x_{t}, h_{t}, z_{t}, m_{t}\right\}_{t=0}^{\infty}$ is a path along which $x_{t}, h_{t}, z_{t}$ and $m_{t}$ are all constant.

From the previous definition and that of $z_{t}$, it follows that along a stationary path consumption and capital grow at the same constant growth rate. Note that no equilibrium condition is imposed in the definition of a stationary path.

Definition $3 A$ balanced growth path (BGP) $\left\{x_{t}, h_{t}, z_{t}, m_{t}\right\}_{t=0}^{\infty}$ is an equilibrium path that is stationary.

Note that from the definition of the variable $m_{t}$, a BGP involves a constant marginal productivity of capital. It is then obvious from the functional form of the production function (3) that a BGP is never reached in finite time when $B>0$ and $\beta>0$. However, we will say that a path $\left\{x_{t}, h_{t}, z_{t}, m_{t}\right\}_{t=0}^{\infty}$ converges to a BGP when

$$
\lim _{t \rightarrow \infty} x_{t}=x, \lim _{t \rightarrow \infty} h_{t}=h, \lim _{t \rightarrow \infty} z_{t}=z, \text { and } \lim _{t \rightarrow \infty} m_{t}=m,
$$

where $x, h, z$ and $m$ are the BGP values of $x_{t}, h_{t}, z_{t}$ and $m_{t}$, respectively.

Let us define the following parameters:

$$
\begin{gathered}
\varepsilon \equiv \frac{\gamma}{1+\rho}, \\
\Delta \equiv \gamma+\sigma(1-\gamma),
\end{gathered}
$$

and

$$
\varphi \equiv \frac{1+A-\delta}{(1+n)(1+\rho)} .
$$

Our next proposition presents necessary conditions to be satisfied by an interior equilibrium path converging towards a BGP with positive growth.

Proposition 1 Let $\varphi>1$. Assume that, for given initial values $z_{0}>0$ and $m_{0}>0$, there is only one strictly positive equilibrium path $\left\{x_{t}, h_{t}, z_{t}, m_{t}\right\}_{t=0}^{\infty}$ and that this path converges to a strictly positive BGP. Then, 
(a) the following conditions are satisfied along the equilibrium path:

$$
\begin{gathered}
z_{t+1}=\left(\frac{z_{t}}{x_{t}}\right)\left(\frac{m_{t}+1-\delta}{1+n}\right)-\frac{1}{1+n}, \\
x_{t+1}=\left(h_{t}\right)^{\frac{1}{1-\sigma}}\left(x_{t}\right)^{\gamma}, \\
m_{t+1}=A+\left(m_{t}-A\right)\left(\frac{1}{1+n}\right)^{\beta-1}\left(m_{t}+1-\delta-\frac{x_{t}}{z_{t}}\right)^{\beta-1},
\end{gathered}
$$

and

$$
\left(\frac{1}{1+\rho}\right)\left(\frac{h_{t}}{x_{t+1}}\right)\left(\frac{1-\theta \varepsilon h_{t+1}}{1-\theta \varepsilon h_{t}}\right)=\frac{1+n}{1+A(1-\beta)+\beta m_{t+1}-\delta}
$$

(b) the strictly positive BGP which the equilibrium path converges to satisfies

$$
\begin{gathered}
x=\varphi^{\frac{1}{\Delta}}>1, \\
m=A, \\
z=\frac{x}{(1+A-\delta)-(1+n) x},
\end{gathered}
$$

and

$$
h=\varphi^{\frac{1-\Delta}{\Delta}} .
$$

Proof. See the Appendix.

Equation (21) tells us that the equilibrium path exhibits sustained growth in the long run. Note that the value of the parameter $\varphi$ is crucial for the existence of positive growth, that is, for $x>1$. Equation (17) follows from the budget constraint and states that it is binding along the equilibrium path. In fact, this equation is the budget constraint defined in terms of the transformed variables. Equation (18) follows from the definition of the transformed variable $h_{t}$ and equation (19) follows from the definition of the transformed variable $m_{t}$. Finally, (20) is the Keynes-Ramsey equation in equilibrium defined in terms of the transformed variables. The left hand side of this equation corresponds to the $M R S_{t, t+1}$ and the right hand side is the $M R T_{t, t+1}$.

Proposition 1 establishes the necessity of equations (17)-(20) in order to obtain a strictly positive equilibrium path converging to a BGP exhibiting sustained growth. If the consumers' maximization problem were convex, these four equations and the corresponding initial and transversality conditions would not be only necessary but also sufficient for obtaining that equilibrium path when $\varphi>1$. Given the assumption of non-increasing returns to scale, the resource constraint (4) defines a convex set of feasible solutions. Thus, the consumers' problem would be convex if the objective function (5) were concave. Stokey et al. (1989, ch. 4) have shown that concavity of the instantaneous utility function is a sufficient, although not necessary, condition that guarantees the concavity of the objective function. However, as follows from our previous discussion, the instantaneous utility function is not concave in this model when $\theta \neq 0$. Therefore, since the convexity of the consumers' maximization problem is not guaranteed, the equilibrium path could be non-interior (i.e., not strictly positive) and, in this case, the system of difference equations (17)-(20) would not characterize that equilibrium path. The following propositions provide conditions aimed at ensuring the 
existence of an interior equilibrium path characterized by the previous dynamic system. We start by imposing a restriction on the values of $\sigma$ compatible with the existence of an interior (i.e., strictly positive) equilibrium path. ${ }^{4}$

Proposition 2 Let $\theta>0$ and assume that there exists a strictly positive equilibrium path $\left\{x_{t}, h_{t}, z_{t}, m_{t}\right\}_{t=0}^{\infty}$ for given $z_{0}>0$ and $m_{0}>0$. Then, $\sigma \geq 1$.

Proof. We proceed by contradiction and assume that $\sigma<1$. In this case, it is easy to check that $u_{t+1}=\infty$ when $c_{t}=0$ and $c_{t+1}>0$, since then $v_{t+1}=0$. Moreover, $u_{t}=0$ when $c_{t}=0$ and $c_{t-1}=\bar{c}_{t-1}>0$. This implies that paths for which consumption and the gross rate $x_{t}$ of consumption are equal to zero in some, but not all, periods deliver higher discounted utility than any strictly positive path. Note that to achieve zero consumption in some periods is always feasible (see (4)).

In view of Proposition 2, if $\sigma<1$ the solution to the dynamic optimization problem cannot be interior when $\theta>0 .{ }^{5}$ The following proposition provides sufficient conditions for an interior equilibrium path:

Proposition 3 Let $\sigma \geq 1$ and $\varphi>1$. Assume that, for all initial values $z_{0}>0$ and $m_{0}>0$, there is only one path $\left\{x_{t}, h_{t}, z_{t}, m_{t}\right\}_{t=0}^{\infty}$ solving the system of difference equations (17)-(20), and that this path is strictly positive and converges to a strictly positive stationary path. Then, the path $\left\{x_{t}, h_{t}, z_{t}, m_{t}\right\}_{t=0}^{\infty}$ is an equilibrium path. Moreover, the stationary path given by expressions (21)-(24) is the unique strictly positive BGP of the economy.

\section{Proof. See the Appendix.}

The previous proposition tells us that, when $\sigma \geq 1$ and $\varphi>1$, an equilibrium path that converges to an interior BGP is fully characterized by the dynamic system composed by the difference equations (17)-(20), together with the initial conditions, for any value of the parameter $\theta$ in the closed interval $[0,1]$. In particular, we will use the previous system of equations to characterize the equilibrium dynamics corresponding to the following two extreme models, which are commonly found in the literature: the IH model and the CJ model. We will see that both models exhibit saddle path stability towards a unique BGP and, thus, the assumptions in Proposition 3 are clearly met. According to the results of this section, we will maintain the assumptions $\sigma \geq 1$ and $\varphi>1$ throughout the rest of the paper.

Concerning the properties of the BGP, note that the stationary rate $x$ of economic growth given in (21) increases with the value of the parameter $A$ measuring total factor productivity (TFP, henceforth) in the long run. The intuition behind this result can be obtained from the Keynes-Ramsey equation (16). From that equation we observe that an increase in TFP reduces the cost of shifting resources to future periods and, thus, drives the price of future consumption in terms of present consumption down. This encourages consumers to shift present consumption to the future and, thus, the rate of economic growth must increase.

\footnotetext{
${ }^{4}$ Note that, if we had assumed that $\gamma<0$, the instantaneous utility function could be concave and the consumers' maximization problem would be convex. In this case, the condition stated in Proposition 2 is not required to guarantee an interior equilibrium path.

${ }^{5}$ Note that the arguments to rule out the case $\sigma<1$ do not apply when the reference variable does not depend on own past consumption $(\theta=0)$. Thus, in the caching-up with the Joneses model, the equilibrium could be characterized by (17)-(20) even if $\sigma<1$.
} 
Note also that, if habits become more important (which amounts to an increase in the value of the parameter $\gamma$ ), then the growth rate goes up when $\sigma>1$. Moreover, the effect on the growth rate of an increase in TFP becomes larger for higher values of $\gamma \cdot{ }^{6}$ This occurs because the introduction of habits makes the intertemporal elasticity of substitution larger and this accelerates economic growth as the ratio of present to past consumption is forced to increase. ${ }^{7}$ This result is in stark contrast to that obtained by Shieh et al. (2000), where the introduction of internal habits could deter growth in some cases. This difference arises because Shieh et al. (2000) did not introduce conditions that guarantee the existence of interior solutions. ${ }^{8}$

\section{Equilibrium under Internal Habits (IH)}

In this section we assume that consumers view only their own past consumption as the standard of living to be used as a reference. Therefore, we impose $\theta=1$ in expression (1) and, thus, the reference variable becomes simply $v_{t}=c_{t-1}$. In this case the KeynesRamsey equation (20) simplifies to

$$
\left(\frac{1}{1+\rho}\right)\left(\frac{h_{t}}{x_{t+1}}\right)\left(\frac{1-\varepsilon h_{t+1}}{1-\varepsilon h_{t}}\right)=\frac{1+n}{1+A(1-\beta)+\beta m_{t+1}-\delta},
$$

where the left hand side of the equation is the marginal rate of substitution in the internal habit formation model ( $M R S_{t, t+1}^{I H}$, henceforth).

Given the initial conditions $m_{0}=f\left(k_{0}\right) / k_{0}$ and $z_{0}=k_{0} / c_{-1}$, we can thus define an interior equilibrium path of the IH model as a strictly positive path $\left\{m_{t}, x_{t}, z_{t}, h_{t}\right\}_{t=0}^{\infty}$ satisfying the difference equations (17), (18), (19) and (25), and the corresponding transversality conditions. The BGP of the IH model is given by the expressions (21)-(24), since these expressions do not depend on the value taken by the parameter $\theta$.

The next two propositions characterize the transitional dynamics of the economic system in the neighborhood of the BGP. This transitional dynamics was already established by Carroll et al. (1997) when the technology is represented by an $A k$ production function. We extend the analysis to the Sobelow production function, where the marginal productivity of capital is time-varying.

Proposition 4 The BGP of the IH model is saddle path stable.

Proof. See the Appendix.

Propositions 3 and 4 allow us to conclude that, for a given pair of initial conditions $z_{0}$ and $m_{0}$ sufficiently close to the stationary values $z$ and $m$, respectively, there is a unique equilibrium path. Moreover, this equilibrium path is the saddle path converging to the BGP.

Proposition 5 Given the initial conditions $z_{0}>0$ and $m_{0}>0$, the following holds for the IH model:

\footnotetext{
${ }^{6}$ Observe that $\frac{\partial x}{\partial \gamma}>0$ and $\frac{\partial x}{\partial A \partial \gamma}>0$ when $\sigma>1$.

${ }^{7}$ Note that, if we define the stationary intertemporal elasticity of substitution as the elasticity of the stationary rate of growth with respect to the asymptotic return to capital, this stationary elasticity is given by the value of $1 / \Delta$. Clearly, this elasticity is strictly increasing in $\gamma$ when $\sigma>1$.

${ }^{8}$ In their paper, the introduction of habits may reduce the long run growth rate because they do not assume that $\sigma \geq 1$. In our paper, this assumption is required to rule out corner solutions yielding unbounded utility.
} 
(a) If $B=0$ then the variables $x_{t}$ and $h_{t}$ will both exhibit a monotonic behavior along the transition towards the BGP. In particular, if $z_{0}<z\left(z_{0}>z\right)$, then the variables $x_{t}$ and $h_{t}$ will increase (decrease) toward their respective stationary values.

(b) If $B>0$ then the variables $x_{t}$ and $h_{t}$ could exhibit a non-monotonic behavior along the transition towards the BGP.

\section{Proof. See the Appendix.}

When $B=0$ the technology is characterized by an $A k$ production function and, hence, $m_{t}=A$. In this case, there is only one state variable, $z_{t}$, and the behavior of $x_{t}$ and $h_{t}$ only depends on the initial value $z_{0}$ of the state variable. However, when $B>0$ there are two state variables, $z_{t}$ and $m_{t}$, and the transition of $x_{t}$ and $h_{t}$ depends on the particular initial values of these two variables. This transition could then be nonmonotonic. Hence, our model could give rise to a transitory non-monotonic behavior of the growth rate. ${ }^{9}$ Therefore, while Carroll et al. (1997) have shown that the consumption growth rate in a model with an $A k$ production function displays a monotonic convergence towards the BGP when preferences are not time-separable, we show that this convergence could be non-monotonic when the production function exhibits diminishing returns to scale. In this case, a reduction in the stock of capital may cause either an increase or a decrease in the consumption growth rate depending on the initial stock of habits. In contrast, Carroll et al. (1997) have shown that a reduction in the stock of capital causes an unambiguous reduction in the consumption growth rate when the production function exhibits constant returns to scale. The intuition behind our result lies in the fact that, when the capital stock becomes smaller, the return on investment increases under diminishing returns to scale and this has a positive effect on the growth rate. However, the reduction in the capital stock makes the amounts of both capital and output small relative to the consumption reference, so that agents would be forced to choose a consumption level so large that it would not be sustainable in the long run. Therefore, such a consumption level will have to decrease in the future. This means that habits make the growth rate decrease as a response to a reduction in the capital stock, while diminishing returns account for the opposite effect. Obviously, these two opposite forces explain both the ambiguity of the response of the growth rate to changes in the stock of capital and the non-monotonic behavior during the transition.

The previous result has also implications for the cross-country convergence. Consider the original model in terms of the variables $c_{t}$ and $k_{t}$. When $B>0$ the transitional dynamics of the growth rate depends on the particular initial values of both the capital level and the reference level of consumption. This means that, under diminishing returns to capital, two economies with different initial capital stocks will follow different equilibrium paths for $x_{t}$ even if they share a common initial value of the ratio $z_{t}$ of capital to consumption reference. On the contrary, when the production function is $A k$, the transitional dynamics of the growth rate $x_{t}$ only depends on the initial value of the ratio $z_{t}$. In this case, two economies with the same initial value of $z_{t}$ will follow equilibrium paths with identical growth rates regardless of their initial levels of capital. It follows that cross-country differences in the growth rate can only be explained by differences in the ratio $z_{t}$ when the production function is $A k$, whereas they can be explained by differences on the values of both the stock of capital and the reference level of consumption when the production function exhibits diminishing returns to scale.

\footnotetext{
${ }^{9}$ In the proof of Proposition 5 we provide an example of an economy exhibiting such a non-monotonic behavior.
} 


\section{Equilibrium under Catching-up with the Joneses (CJ)}

In this section we make $\theta=0$ in expression (1). This means that the average aggregate consumption of the previous period is now the reference level of consumption, that is, $v_{t}=\bar{c}_{t-1}$. Therefore, the model displays the typical "catching-up with the Joneses" feature, since average past consumption enters into the consumers' utility as a negative externality.

We next derive the equations characterizing the dynamic equilibrium of this particular model. Since $\theta=0$, the Keynes-Ramsey equation (20) is simply

$$
\left(\frac{1}{1+\rho}\right)\left(\frac{h_{t}}{x_{t+1}}\right)=\frac{1+n}{1+A(1-\beta)+\beta m_{t+1}-\delta},
$$

where the left hand side of equation (26) is the marginal rate of substitution in the catching-up with the Joneses model $\left(M R S_{t, t+1}^{C J}\right.$, henceforth). Using the definition of $h_{t}$ introduced in Section 2, (26) becomes

$$
x_{t+1}=x_{t}^{\frac{\gamma(\sigma-1)}{\sigma}}\left(\frac{1+A(1-\beta)+\beta m_{t+1}-\delta}{(1+n)(1+\rho)}\right)^{\frac{1}{\sigma}} .
$$

In contrast to the IH model, the equilibrium is now fully described by only three variables: $z_{t}, m_{t}$, and $x_{t}$. The first two variables are the state variables, whereas the third one is the control variable. Hence, given the initial conditions $m_{0}=f\left(k_{0}\right) / k_{0}$ and $z_{0}=k_{0} / c_{-1}$, we define an equilibrium path of the CJ model as a strictly positive path $\left\{m_{t}, x_{t}, z_{t}\right\}_{t=0}^{\infty}$, satisfying the difference equations (17), (19) and (27), and the corresponding transversality conditions. A BGP will be thus an equilibrium path along which the variables $m_{t}, x_{t}$ and $z_{t}$ are constant. Obviously the gross rate of growth $h_{t}$ of the instantaneous utility $u$ is also constant along a BGP. It is thus clear from the expressions appearing in part (b) of Proposition 1 that the BGP of the CJ model is the same as that of the IH model, since the BGP of the general model of Section 2 is independent of the parameter $\theta$.

We next discuss the intuition for obtaining identical stationary solutions for both models. In the CJ model consumers do not internalize the spillovers accruing from the average past consumption. On the contrary, consumers in the IH model take into account the future effects of their current decisions on consumption. This difference translates into differences between the marginal rate of substitution of both models during the transition, as one can easily see by comparing equations (25) and (26). However, since the discounted sum of utilities is bounded, the growth rates of both instantaneous utility and consumption must converge to a constant value. Hence, it is immediate to see from (25) and (26) that the marginal rates of substitution of both models coincide along a stationary path (i.e., when $x_{t}$ and $h_{t}$ are constant for all $t$ ). The next two propositions characterize the transitional dynamics of the CJ model in the neighborhood of the BGP:

Proposition 6 The BGP of the CJ model is saddle path stable.

Proof. See the Appendix.

The previous result establishes that the equilibrium path of the CJ model is unique for a given pair of initial conditions $z_{0}$ and $m_{0}$ sufficiently close to their respective stationary values $z$ and $m$. Moreover, the equilibrium path converges to the unique BGP. 
Proposition 7 Given the initial conditions $z_{0}>0$ and $m_{0}>0$, the following holds for the CJ model:

(a) If $B=0$ then the variable $x_{t}$ will exhibit a monotonic behavior along the transition towards the BGP. In particular, if $z_{0}<z\left(z_{0}>z\right)$, then the variable $x_{t}$ will increase (decrease) toward its stationary values.

(b) If $B>0$ then the variable $x_{t}$ could exhibit a non-monotonic behavior along the transition towards the BGP.

Proof. See the Appendix.

Proposition 7 has the same qualitative implications for the transitional dynamics and the cross-country convergence of the CJ model as those established by Proposition 5 for the IH model. In other words, the policy functions tracing out the relationship between the state variables and the optimal value of the control variables in the CJ model are qualitatively similar to those of the IH model. However, the efficiency analysis of the next section will show that the relationship between state and control variables differs quantitatively from one model to the other. ${ }^{10}$

\section{$5 \quad$ Efficiency and Optimal Policy}

The equilibrium of the CJ model could be inefficient because consumers do not internalize the spillover effects from average past consumption. This source of inefficiency has been studied by Ljungqvist and Uhlig (2000) in a model without capital accumulation. ${ }^{11}$ In this section, we extend the efficiency analysis into a growth model with capital accumulation. To this end, note that the equilibrium of the IH model described in Section 3 coincides with the solution of the CJ model that a benevolent social planner would implement, since that planner would take into account all the external effects accruing from average past consumption. This means that, in order to deal with efficiency issues, we just have to compare the equilibrium solution of the CJ model with that of the IH model.

The only difference between the equations that characterize the equilibrium paths of the two models lies in the $M R S_{t, t+1}$ appearing in the left hand side of the Keynes-Ramsey equations (25) and (26). Efficiency of the competitive solution of the CJ model requires that the $M R S_{t, t+1}$ obtained in the two models be identical, i.e., $M R S_{t, t+1}^{C J}=M R S_{t, t+1}^{I H}$, where $M R S_{t, t+1}^{I H}$ is the efficient $M R S_{t, t+1}$. Given our assumptions on preferences, the previous efficiency condition is obviously satisfied when $\sigma=1$. However, if $\sigma>1$, then the efficiency condition becomes simply $h_{t+1}=h_{t}$. This equality holds along the BGP, which implies that the equilibrium of the CJ model is asymptotically efficient. This is consistent with the fact that the CJ and IH models share the same BGP, as shown in the previous sections. Finally, the dynamic equilibrium of the CJ model is obviously inefficient during the transition when $\sigma>1$.

In what follows we will show that efficiency can be restored in the CJ model by means of an appropriate tax policy. We present two alternative tax instruments that

\footnotetext{
${ }^{10}$ In the case where the production function is $A k$, Carroll et al. (1997) show that the slope of the equilibrium saddle path (or the policy function) in the CJ model differs from that of the IH model.

${ }^{11}$ Fisher and Hof (2000) have also analyzed equilibrium efficiency in a neoclassical growth model when the source of inefficiency is an externality arising from average current consumption. In contrast, in our paper the externality is associated to the average past consumption level and the results on efficiency are in stark contrast with those obtained by Fisher and Hof (2000). Actually, Ljungqvist and Uhlig (2000) have stressed the fact that inefficiency depends on the timing of the consumption externality.
} 
make identical the two Keynes-Ramsey equations (25) and (26): a tax on net income (or net output) and a tax on consumption. By using a procedure similar to that of Fisher and Hof (2000) and Alonso-Carrera et al. (2001), we derive the corresponding optimal tax rates. We assume that the tax revenues are returned to consumers through a lumpsum subsidy. This assumption implies that the resource constraint of a representative dynasty becomes now

$$
\left(1+\tau_{t}^{c}\right) c_{t}+(1+n) k_{t+1}-k_{t}=\left(1-\tau_{t}^{y}\right)\left[f\left(k_{t}\right)-\delta k_{t}\right]+T_{t}
$$

where $\tau^{c}$ and $\tau^{y}$ are the tax rates on consumption and on net income, respectively, and $T_{t}$ is the lump-sum subsidy that satisfies the following government budget constraint: ${ }^{12}$

$$
T_{t}=\tau_{t}^{y}\left[f\left(k_{t}\right)-\delta k_{t}\right]+\tau_{t}^{c} c_{t} .
$$

Combining (28) and (29) and using the transformed variables, we obtain the resource constraint. Therefore, because all the tax revenue is returned to the consumers as a lump-sum subsidy, the introduction of taxes only modifies the Keynes-Ramsey equation (26). Thus, this equation becomes

$$
\left(\frac{1}{1+\rho}\right)\left(\frac{h_{t}^{C J}}{x_{t+1}^{C J}}\right)=\left(\frac{1+\tau_{t+1}^{c}}{1+\tau_{t}^{c}}\right)\left(\frac{1+n}{1+\left(1-\tau_{t+1}^{y}\right)\left[(1-\beta) A+\beta m_{t+1}^{C J}-\delta\right]}\right)
$$

where the superscript $C J$ is used to denote the variables of the CJ model. Note that the LHS of the previous equation is the marginal rate of substitution $M R S_{t, t+1}^{C J}\left(h_{t}^{C J}, x_{t+1}^{C J}\right)$ of the CJ model. Evaluating (30) at the efficient equilibrium path, and dividing the resulting equation by the Keynes-Ramsey equation (25) of the IH model, we obtain the following optimal taxation condition:

$$
\frac{M R S_{t, t+1}^{C J}\left(h_{t}^{I H}, x_{t+1}^{I H}\right)}{M R S_{t, t+1}^{I H}\left(h_{t+1}^{I H}, h_{t}^{I H}, x_{t+1}^{I H}\right)}=\left(\frac{1+\hat{\tau}_{t+1}^{c}}{1+\hat{\tau}_{t}^{c}}\right)\left(\frac{1+(1-\beta) A+\beta m_{t+1}^{I H}-\delta}{1+\left(1-\hat{\tau}_{t+1}^{y}\right)\left[(1-\beta) A+\beta m_{t+1}^{I H}-\delta\right]}\right),
$$

where the superscript $I H$ is used to denote the equilibrium value of the variables in the IH model; $\hat{\tau}_{t}^{c}$ and $\hat{\tau}_{t}^{y}$ are the optimal values of the tax rates on consumption and income, respectively; and $M R S_{t, t+1}^{I H}\left(h_{t+1}^{I H}, h_{t}^{I H}, x_{t+1}^{I H}\right)$ and $M R S_{t, t+1}^{C J}\left(h_{t}^{I H}, x_{t+1}^{I H}\right)$ are the $M R S_{t, t+1}$ corresponding to the IH model and the CJ model, respectively, when they are evaluated along the efficient equilibrium path. We see from (31) that optimal taxes display time-varying rates off the BGP, while the optimal consumption tax rate is constant and the optimal income tax rate is zero at the BGP. This result about the optimal income tax rate in the long run resembles those obtained by Judd (1985) and Chamley (1986) in models with standard preferences. ${ }^{13}$ Thus, we see that the introduction of habit formation affects the optimal tax rates only during the transition.

If the $M R S_{t, t+1}$ of the CJ model evaluated along the efficient consumption path turns out to be smaller than the efficient $M R S_{t, t+1}$ along the same path, then the

\footnotetext{
${ }^{12}$ Note that a tax on income has the same effects on capital accumulation as a tax on capital income provided the tax revenue is entirely returned to consumers through a lump-sum transfer. Even if the amount of taxes collected for a given tax rate is not the same, the marginal productivity of capital is modified identically under the two tax schemes.

${ }^{13}$ It should be pointed out that in the papers of Judd and Chamley the government intends to finance optimally a given stream of spending. However, in our model the government just uses optimal taxes aimed at correcting for the ineffciencies brought about by consumptions spillovers.
} 
consumers' willingness to shift present consumption to the future will be too small. In this case, condition (31) tells us that the efficient path can be reached by means of either subsidizing output or introducing a tax on consumption with $\hat{\tau}_{t}^{c}>\hat{\tau}_{t+1}^{c}$. These fiscal policies correct the inefficiency because they make present consumption purchases more expensive than the future ones and, hence, they encourage consumers to postpone consumption. A decreasing sequence of tax rates on consumption directly drives the after-tax price of future consumption in terms of present consumption down. Moreover, a subsidy on output also reduces the relative price of future consumption because this policy reduces the cost of shifting resources to future periods. Therefore, if the $M R S_{t, t+1}$ of the CJ model along an efficient path is larger (smaller) than the efficient $M R S_{t, t+1}$, then a welfare-maximizing government must impose either a tax (subsidy) on income or a tax on consumption with a rate that rises (falls) over time.

Finally, we can also characterize the dynamic behavior of the optimal tax rates by expressing them as functions of the state variables of the model. As a first step towards this goal, we show that both rates depend only on the efficient value $h_{t}^{I H}$ of the utility growth rate. On the one hand, making $\hat{\tau}_{t+1}^{y}=0$ and from the definition of the variable $h_{t}^{I H}$, condition (31) can be rewritten as follows:

$$
\frac{\hat{\tau}_{t+1}^{c}-\hat{\tau}_{t}^{c}}{1+\hat{\tau}_{t}^{c}}=\varepsilon\left(\frac{h_{t+1}^{I H}-h_{t}^{I H}}{1-\varepsilon h_{t+1}^{I H}}\right)
$$

We thus see that the optimal tax on consumption increases (decreases) when $h_{t}^{I H}$ increases (decreases). Also note that $\hat{\tau}_{t+1}^{c}=\hat{\tau}_{t}^{c}$ along the BGP. Therefore, any sequence of constant tax rates (not necessarily equal to zero) on consumption is optimal along a BGP.

On the other hand, imposing $\hat{\tau}_{t}^{c}=0$ for all $t$ in condition (31), we obtain that the optimal rate of the income tax is

$$
\hat{\tau}_{t+1}^{y}=\varepsilon\left(\frac{h_{t+1}^{I H}-h_{t}^{I H}}{1-\varepsilon h_{t}^{I H}}\right)\left(\frac{1+(1-\beta) A+\beta m_{t+1}^{I H}-\delta}{(1-\beta) A+\beta m_{t+1}^{I H}-\delta}\right) .
$$

Note that this optimal rate equals zero along the BGP as $h_{t+1}^{I H}=h_{t}^{I H}$. However, this tax is positive when the growth rate of utility increases with time, $h_{t+1}^{I H}>h_{t}^{I H}$, and it is negative otherwise.

We have then shown that the evolution of the optimal rates of both taxes is qualitatively determined only by the transition of the variable $h_{t}^{I H}$ along the efficient equilibrium. This occurs because, when habits are modeled in a multiplicative way, there is a direct relation between the variable $h_{t}^{I H}$ being increasing (decreasing) along the transition and the $M R S_{t, t+1}$ being suboptimally large (small). Proposition 5 describes the behavior of $h_{t}^{I H}$ during the transition, so that we can derive the evolution of the optimal tax rates directly from this Proposition.

Corollary 1 (a) The sequence of optimal consumption tax rates $\left\{\hat{\tau}_{t}^{c}\right\}_{t=1}^{\infty}$ around the $B G P$ could be either monotonic or non-monotonic for a given arbitrary value of $\hat{\tau}_{0}^{c}$. This sequence converges to a constant.

(b) The sequence of optimal income tax rates $\left\{\hat{\tau}_{t}^{y}\right\}_{t=1}^{\infty}$ around the BGP could either exhibit the same sign or change its sign. This sequence converges to zero.

Proof. Obvious from Proposition 5 and expressions (32) and (33). 
Part (a) of Proposition 5 tells us that if $z_{0}<z$, then $h_{t}^{I H}$ will increase. In this case, the $M R S_{t, t+1}$ will be suboptimally large and the optimal tax policy will consist on either an increasing sequence of tax rates on consumption or a positive tax rate on income. The opposite will occur if $z_{0}>z$. However, Part (b) of Proposition 5 tells us that, if the marginal productivity of capital is not constant, namely, when $B>0$, then the variable $h_{t}^{I H}$ could exhibit a non-monotonic behavior along the transition. This implies that the optimal consumption tax could grow during a number of periods and decrease afterwards. Similarly, the optimal tax on income could be positive during some periods and become negative later on, or vice versa.

When $B>0$ there are two state variables, $z_{t}$ and $m_{t}$, and the transition of the variable $h_{t}^{I H}$ depends on the initial value of these two variables. Therefore, the optimal fiscal policy depends also on the initial values of these two state variables or, equivalently, on the initial values of both capital and the reference level of consumption. This means that, under strictly decreasing returns to capital, two economies with different initial capital stocks will have different optimal tax rates even if they share a common initial value of the ratio $z_{t}$ of capital to consumption reference. On the contrary, when the production function is $A k$, the optimal fiscal policy only depends on the initial value of the ratio $z_{t}$. In this case, two economies with the same initial value of $z_{t}$ will exhibit the same optimal tax rates regardless of their initial levels of capital.

We can provide a numerical example to compare the optimal income taxes for two economies that are identical except on the initial value of the state variable $m_{t}$. Let us assume that the parameters characterizing both economies take the following values: ${ }^{14}$

$$
A=0.183, \sigma=5, \delta=0.09, n=0, \rho=0.03, \gamma=0.5 \text {, and } \beta=0.2 \text {. }
$$

Both economies share the same initial value of the ratio of capital to consumption reference, namely, $z_{0}=0.99 z$. We also assume that in one economy $m_{0}=m$ and, since $m=A$, the technology is characterized by an $A k$ production function from $t=0 \mathrm{on}$. In the other economy we set $m_{0}=1.01 \mathrm{~m}$ and, hence, capital exhibits strictly decreasing returns to capital. The optimal rate of the income tax when the technology is $A k$ turns out to be always positive along the transition and converges to zero. However, the optimal tax rate on income when the production function exhibits diminishing returns to scale takes negative values for $t \leq 6$, while for $t>6$ it takes positive values. Similarly, under diminishing returns to scale the tax rate on consumption is decreasing for $t \leq 6$ and increasing for $t>6$ for any arbitrarily given initial tax rate. Note that, when $t=6.7081$, it holds that $h_{t+1}^{I H}=h_{t}^{I H}$, which is consistent with the expressions (32) and (33) characterizing optimal tax rates. This numerical example has thus illustrated clearly the potential non-monotonicity of optimal tax rates when the technology exhibits strictly decreasing returns to capital.

The previous results on the optimal income tax rate are in a stark contrast with the results obtained by Ljungqvist and Uhlig (2000) in a catching-up with the Joneses model without capital accumulation. These authors show that the optimal income tax rate is positive when there is a high realization of a productivity shock raising the growth rate, and it is negative otherwise. In this paper we show that this result does not hold when capital accumulation is introduced. On the one hand, if the production function exhibits

\footnotetext{
${ }^{14}$ We set the values of $\delta, n, \rho, \sigma, \gamma$ as in Caroll et al. (2000). In particular, the values of $\sigma$ and $\gamma$ are such that the inverse of the stationary intertemporal elasticity of substitution takes the reasonable value $\Delta=3$ (see footnote 7 ), and the value of $A$ is such that yields a long-run growth rate equal to $2 \%$. The value of $\beta$ allows us to obtain a speed of convergence of $1.6 \%$. This configuration of parameter values is also used in the proof of Proposition 5 (see (46) in the Appendix).
} 
constant returns to scale, the optimal income tax rate will take negative values when the consumption growth rate is above its BGP value and takes positive values otherwise. On the other hand, if the production function exhibits diminishing returns to scale, the optimal income tax rate will be either procyclical or countercyclical, depending on the reference level of consumption. Thus, we conclude that the results on optimal fiscal policy obtained in a model without capital do not hold in a model exhibiting capital accumulation.

\section{Conclusion}

We have analyzed the dynamic equilibrium of an endogenous growth model where preferences are time-dependent. In particular, we have assumed the existence of internal and external habit formation in consumption. Thus, utility depends on own consumption relative to a reference level, which grows with both past own consumption and past average consumption. The presence of internal habits makes the instantaneous utility function non-concave and, hence, concavity of the objective function is not guaranteed. We have provided conditions under which the equilibrium path is the solution to a dynamic system formed by standard first order conditions. We have then studied the equilibrium of two growth models, namely, the IH model, where the reference is the own past consumption, and the CJ model, where the reference takes the form of an externality accruing from average past consumption.

The introduction of a consumption externality makes the equilibrium of the CJ model inefficient during the transition. We have then characterized the optimal fiscal policy. In particular, we have derived the optimal tax rates on income and on consumption. The optimal tax rate on income is zero along the BGP, whereas it is different from zero along the transition. The optimal rate of consumption tax is constant at the BGP, while it could either increase or decrease with time along the transition. We have shown how the value of optimal tax rates during the transition depends on the initial values of both the reference variable and capital. More precisely, if the marginal productivity of capital is constant, then the optimal tax rates will only depend on the initial value of the ratio of capital to consumption reference. However, if that marginal productivity is not constant during the transition, then the optimal tax rates will depend on the initial values of both capital and consumption and they could exhibit a non-monotonic dynamics. 


\section{References}

[1] Abel, A., (1990). "Asset Prices under Habit Formation and Catching up with the Joneses," American Economic Review 80, 38-42.

[2] Abel, A., (1999). "Risk Premia and Term Premia in General Equilibrium," Journal of Monetary Economics 43: 3-33.

[3] Alonso-Carrera, J, J. Caballé, and X. Raurich, (2001). "Consumption Externalities, Habit Formation, and Equilibrium Efficiency," UAB-IAE Working Paper 499.01.

[4] Carroll, C., (2000). "Solving Consumption Models with Multiplicative Habits," Economics Letters 68: 67-77.

[5] Carroll, C., J. Overland, and D. Weil, (1997). "Comparison Utility in a Growth Model," Journal of Economic Growth 2: 339-367.

[6] Carroll, C., J. Overland, and D. Weil, (2000). "Saving and Growth with Habit Formation," American Economic Review 90: 1-15.

[7] Chamley, C., (1986). "Optimal Taxation of Capital Income in General Equilibrium with Infinite Lives," Econometrica 54: 607-22.

[8] Fisher, W., and F. Hof, (2000). "Relative Consumption, Economic Growth, and Taxation," Journal of Economics 72: 241-262.

[9] Fuhrer, J.C., (2000). "Habit Formation in Consumption and its Implications for Monetary Policy," American Economic Review 90: 367-90.

[10] Galí, J., (1994). "Keeping up with the Joneses: Consumption Externalities, Portfolio Choice, and Asset Prices," Journal of Money Credit and Banking 26: 1-8.

[11] Jones, L., and R. Manuelli, (1990). "A Convex Model of Economic Growth: Theory and Policy Implications," Journal of Political Economy 98: 1008-1038.

[12] Judd, K., (1985). "Redistributive Taxation in a Simple Perfect Foresight Model," Journal of Publics Economics 28: 59-83.

[13] Lettau, M., and H. Uhlig, (2000). "Can Habit Formation Be Reconciled with Business Cycles Facts?," Review of Economic Dynamics 3: 79-99.

[14] Ljungqvist, L., and H. Uhlig, (2000). "Tax Policy and Aggregate Demand Management Under Catching Up with the Joneses," American Economic Review 90: 356-366.

[15] Shieh, J., C. Lai, and W. Chang, (2000). "Addictive Behaviour and Endogenous Growth," Journal of Economics 72: 263-273.

[16] Stokey, N., R. Lucas, and E. Prescott, (1989). Recursive Methods in Economic Dynamics, Harvard University Press. 


\section{A Appendix}

\section{Proof of Proposition 1.}

(a) We use the transformed variables $x_{t}$ and $h_{t}$, and the equilibrium condition $c_{t}=\bar{c}_{t}$, in order to rewrite equation (16) as follows

$$
\left(\frac{1}{1+\rho}\right)\left(\frac{h_{t}}{x_{t+1}}\right)\left(\frac{1-\theta \varepsilon h_{t+1}}{1-\theta \varepsilon h_{t}}\right)=\frac{1+n}{1+f^{\prime}\left(k_{t+1}\right)-\delta} .
$$

Note that, by using the transformed variable $m_{t}$, the marginal product of capital is then

$$
f^{\prime}\left(k_{t+1}\right)=A(1-\beta)+\beta m_{t+1} .
$$

Hence, after rearranging terms, equation (34) becomes equation (20) in the statement of the proposition.

Evaluating equation (20) when $t$ tends to infinity, and using the definitions of $h_{t}$ and $x$, and the functional form of the utility function, we get

$$
x^{-[\gamma+\sigma(1-\gamma)]}=\lim _{t \rightarrow \infty} \frac{(1+n)(1+\rho)}{1+A(1-\beta)+\beta m_{t+1}-\delta} .
$$

Since $\gamma+\sigma(1-\gamma)>0$ and

$$
\lim _{t \rightarrow \infty} \frac{(1+n)(1+\rho)}{1+A(1-\beta)+\beta m_{t+1}-\delta}=\frac{(1+n)(1+\rho)}{1+A-\delta}=\frac{1}{\varphi}<1,
$$

it follows from (35) that (21) holds.

Since $x>1$ implies that $\lim _{t \rightarrow \infty} c_{t}=\infty$ and $c_{t}=\bar{c}_{t}$, we conclude from (10) that (9) holds with equality. Therefore,

$$
\begin{gathered}
\lim _{t \rightarrow \infty} \lambda_{t}=\lim _{t \rightarrow \infty}\left[\left(\frac{1}{1+\rho}\right)^{t} \frac{\partial u_{t}}{\partial c_{t}}\right]=(1-\sigma) \lim _{t \rightarrow \infty}\left[\left(\frac{1}{1+\rho}\right)^{t} \frac{u_{t}}{c_{t}}\right]= \\
x^{\gamma(1-\sigma)} \lim _{t \rightarrow \infty}\left[\left(\frac{1}{1+\rho}\right)^{t}\left(c_{t}\right)^{-[\gamma+\sigma(1-\gamma)]}\right]=0,
\end{gathered}
$$

as $\gamma+\sigma(1-\gamma)>0$. Rewriting (11), we get

$$
\lambda_{t} \geq\left[\frac{1+f^{\prime}\left(k_{t+1}\right)-\delta}{1+n}\right] \lambda_{t+1} .
$$

Since $1+f^{\prime}\left(k_{t+1}\right)-\delta \geq 1+A-\delta>(1+n)(1+\rho)>1+n$, we have that $\lambda_{t}>\lambda_{t+1}$, which together with (36), implies that $\lambda_{t}>0$ for all $t$ finite. Therefore, (15) implies that the resource constraint (4) is satisfied with equality and, by using the transformed variables, we get equation (17).

From the definition of $h_{t}$ and the functional form of the utility function $u_{t}$, it follows that equation (18) must also hold. Combining the definition of $m_{t}$ with the functional form of $f\left(k_{t+1}\right)$ and the resource constraint (17), we obtain equation (19).

(b) We have already proved that (21) holds. From the definition of the variable $z_{t}$, we have that $\lim _{t \rightarrow \infty}\left(k_{t+1} / k_{t}\right)=x>1$ and, thus, $\lim _{t \rightarrow \infty} m_{t}=A$ (see (3)). The stationary values $z$ and $h$ are obtained from a direct computation aimed at obtaining their limiting values according to the dynamic system formed by equations (17)-(20). 
Proof of Proposition 3. First, note that the unique strictly positive stationary path solving the system of difference equations (17)-(20) is given by expressions (21)-(24), as can be seen by making $m_{t}=m>0, x_{t}=x>0, z_{t}=z>0$ and $h_{t}=h>0$ for all $t$ in that dynamic system and solving for the corresponding stationary values.

Let us check that the stationary path solving both the Keynes-Ramsey equation (16) and the resource constraint (4) with equality satisfies all the first order conditions and transversality conditions for optimality. By defining $\lambda_{t}$ as

$$
\lambda_{t}=\left(\frac{1}{1+\rho}\right)^{t} \frac{\partial u_{t}}{\partial c_{t}}+\left(\frac{1}{1+\rho}\right)^{t+1} \frac{\partial u_{t+1}}{\partial v_{t+1}} \frac{\partial v_{t+1}}{\partial c_{t}}
$$

the necessary first order conditions $(7),(8),(11),(12)$, and (15) are automatically satisfied by any path solving both (16) and the resource constraint (4) with equality. Moreover, from the same arguments appearing in the proof of Proposition 1, it is immediate to see that $\lambda_{t} \geq 0$, with strict inequality for all $t$ finite.

Let us now check that the stationary solution to the dynamic system formed by equation (16) and the resource constraint (4) with equality satisfies also the transversality conditions (9), (10), (13), and (14) when $\sigma \geq 1$ and $x>1$. Using (37), the transversality condition (10) becomes

$$
\lim _{t \rightarrow \infty}\left\{\left[\left(\frac{1}{1+\rho}\right)^{t+1} \frac{\partial u_{t+1}}{\partial v_{t+1}} \frac{\partial v_{t+1}}{\partial c_{t}}\right] \cdot c_{t}\right\}=0,
$$

which, making $c_{t}=\bar{c}_{t}$, is equal to

$$
-\gamma \theta(1-\sigma) \lim _{t \rightarrow \infty}\left\{\left(\frac{1}{1+\rho}\right)^{t+1} u_{t+1}\right\}=0 .
$$

Moreover, at the stationary path, we have that $u_{t+1} / u_{t}=x^{(1-\gamma)(1-\sigma)}$ and, as $x>1$, $\gamma \in(0,1)$ and $\sigma \geq 1$, it follows that

$$
\frac{u_{t+1}}{u_{t}}<1<1+\rho .
$$

Therefore, from (40) we can conclude that (39) effectively holds, so that (10) is fulfilled. Moreover, using (37), (9) could also be written as

$$
-\gamma \theta x^{1-\sigma} \lim _{t \rightarrow \infty}\left\{\left(\frac{1}{1+\rho}\right)^{t+1}\left(c_{t}\right)^{-[\gamma+\sigma(1-\gamma)]}\right\} \leq 0,
$$

which is satisfied with equality as $\lim _{t \rightarrow \infty} c_{t}=\infty$ and $\gamma+\sigma(1-\gamma)>0$.

Now, we can use (36) to conclude that (13) also holds with equality. Moreover, using again (36), the transversality condition (14) becomes

$$
-(1+n) x^{\gamma(1-\sigma)} \lim _{t \rightarrow \infty}\left(\frac{1}{1+\rho}\right)^{t}\left(c_{t}\right)^{(1-\gamma)(1-\sigma)} \frac{k_{t+1}}{c_{t}}=0 .
$$

Recall that

$$
\lim _{t \rightarrow \infty} \frac{k_{t+1}}{c_{t}}=z=\frac{x}{(1+A-\delta)-(1+n) x} .
$$

Therefore, since $\sigma \geq 1$ and $\lim _{t \rightarrow \infty} c_{t}=\infty$, we conclude that (41) effectively holds, so that (14) is satisfied. 
We have thus proved that all the necessary conditions for optimality are satisfied by the unique stationary path solving the dynamic system formed by (16) and the resource constraint (4) with equality. Note that this means that the necessary conditions for optimality are satisfied by the unique stationary path $\left\{x_{t}, h_{t}, z_{t}, m_{t}\right\}_{t=0}^{\infty}$ solving the system of equations (17)-(20) for given initial values $z_{0}>0$ and $m_{0}>0$.

By assumption, there is only one path $\left\{x_{t}, h_{t}, z_{t}, m_{t}\right\}_{t=0}^{\infty}$ satisfying the necessary conditions for optimality (17)-(20) and, moreover, this path is strictly positive and converges to a strictly positive BGP. Furthermore, we have just proved that this BGP satisfies all the transversality conditions. Therefore, we only have to prove that noninterior paths deliver lower levels of utility in order to conclude that the proposed interior path $\left\{x_{t}, h_{t}, z_{t}, m_{t}\right\}_{t=0}^{\infty}$ is the unique equilibrium path of the economy. To this end, let us express the budget constraint (4) in terms of the transformed variables,

$$
z_{t+1} \leq\left(\frac{z_{t}}{x_{t}}\right)\left(\frac{m_{t}+1-\delta}{1+n}\right)-\frac{1}{1+n} .
$$

For given values of the state variables $z_{t}>0$ and $m_{t}>0$ in period $t$, the growth rate $x_{t}$ of consumption must satisfy the following feasibility condition:

$$
0 \leq x_{t} \leq z_{t}\left(m_{t}+1-\delta\right),
$$

as follows from combining (42) with the fact that $z_{t+1} \geq 0$. On the one hand, the discounted sum of utilities is equal to $-\infty$ when $x_{t}=0$, since $\sigma \geq 1$. On the other hand, if $x_{t}=z_{t}\left(m_{t}+1-\delta\right)$, then the constraint (42) implies that $z_{t+1}=0$, which in turn implies that $x_{t+1}=0$ (see (43)). Again, this results in a discounted sum of utilities equal to $-\infty$ when $\sigma \geq 1$. Therefore, optimality requires that $x_{t} \in\left(0, z_{t}\left(m_{t}+1-\delta\right)\right)$ and, hence, $h_{t} \in(0, \infty)$ for all $t$. We have thus ruled out non-interior values for the control variables, since their corresponding paths yield a level of utility that is strictly dominated by the one associated with an arbitrary interior path converging to a strictly positive stationary path.

Proof of Proposition 4. Using (17), (18), (19) and (25), we obtain the following Jacobian matrix evaluated at the BGP:

$$
M^{I H} \equiv\left(\begin{array}{cccc}
\frac{\partial x_{t+1}}{\partial x_{t}} & \frac{\partial x_{t+1}}{\partial z_{t}} & \frac{\partial x_{t+1}}{\partial m_{t}} & \frac{\partial x_{t+1}}{\partial h_{t}} \\
\frac{\partial z_{t+1}}{\partial x_{t}} & \frac{\partial z_{t+1}}{\partial z_{t}} & \frac{\partial z_{t+1}}{\partial m_{t}} & \frac{\partial z_{t+1}}{\partial h_{t}} \\
\frac{\partial m_{t+1}}{\partial x_{t}} & \frac{\partial m_{t+1}}{\partial z_{t}} & \frac{\partial m_{t+1}}{\partial m_{t}} & \frac{\partial m_{t+1}}{\partial h_{t}} \\
\frac{\partial h_{t+1}}{\partial x_{t}} & \frac{\partial h_{t+1}}{\partial z_{t}} & \frac{\partial h_{t+1}}{\partial m_{t}} & \frac{\partial h_{t+1}}{\partial h_{t}}
\end{array}\right)=\left(\begin{array}{cccc}
\gamma & 0 & 0 & \frac{\varphi}{1-\sigma} \\
-\frac{(1+A-\delta) z}{(1+n) x^{2}} & \frac{1+A-\delta}{(1+n) x} & \frac{z}{(1+n) x} & 0 \\
0 & 0 & x^{\beta-1} & 0 \\
-\frac{(1-\varepsilon h) \gamma}{\varepsilon h \varphi} & 0 & \frac{x^{\beta-1}(1-\varepsilon h) \beta}{(1+n)(1+\rho) \varepsilon \varphi} & \frac{\varepsilon h-\sigma}{(1-\sigma) \varepsilon h}
\end{array}\right) .
$$

It is immediate to see that the eigenvalues $\mu_{n}, n=1,2,3,4$, of $M^{I H}$ are $\mu_{1}=x^{\beta-1}$, $\mu_{2}=\frac{1+A-\delta}{(1+n) x}$ and the two roots $\mu_{3}$ and $\mu_{4}$ solving the equation $Q(\mu)=0$, where

$$
Q(\mu)=\mu^{2}-\left[1+\gamma+\left(\frac{\sigma}{\sigma-1}\right)\left(\frac{1-\varepsilon h}{\varepsilon h}\right)\right] \mu+\frac{1+\rho}{h} .
$$

Note that $\mu_{1} \in(0,1)$ because $x>1$. From the resource constraint $(17)$, we see that $\mu_{2}=1+\frac{1}{(1+n) z}>1$. Finally, in order to determine the value of $\mu_{3}$ and $\mu_{4}$, we must first characterize the function $Q(\mu)$. This function is a convex function with $Q(0)>0$ and

$$
Q(1)=\left[\frac{\sigma(\gamma-1)-\gamma}{\sigma-1}\right]\left(\frac{1-\varepsilon h}{\varepsilon h}\right)
$$


Condition $\varphi>1$ implies that $\varepsilon h<1$. Hence, $Q(1)$ is negative and finite when $\sigma>1$, whereas it converges to minus infinite as $\sigma$ tends to one.

From our previous analysis we can conclude that, if $\sigma>1$, then $\mu_{3}>1>\mu_{4}>0$. Therefore, all four eigenvalues are positive, two eigenvalues are smaller than one, and two eigenvalues are larger than one. This means in turn that the BGP exhibits saddle path stability, since the IH model has two state variables, $z_{t}$ and $m_{t}$.

When $\sigma=1$, the system of equations defining the equilibrium path is formed just by equations (17), (19) and (25), and the equilibrium is fully described without the variable $h_{t}$. In this case, $Q(\mu)$ has a unique root, which is $\mu_{3}=0$. This means that the equilibrium also exhibits saddle path stability in this case.

Proof of Proposition 5. Using Proposition 4, we obtain the following equations characterizing the equilibrium saddle path around the BGP for the IH model:

$$
q_{t}=E^{1} \mu_{1}^{t}+E^{4} \mu_{4}^{t}+q
$$

where $q_{t}=\left(h_{t}, x_{t}, m_{t}, z_{t}\right), \quad \mu_{1}$ and $\mu_{4}$ are the stable eigenvalues, and $E^{1}=\left(E_{h}^{1}, E_{x}^{1}, E_{m}^{1}, E_{z}^{1}\right)$ and $E^{4}=\left(E_{h}^{4}, E_{x}^{4}, E_{m}^{4}, E_{z}^{4}\right)$ are the eigenvectors associated with the eigenvalues $\mu_{1}$ and $\mu_{4}$, respectively. We proceed to find some properties of the eigenvectors $E^{1}$ and $E^{4}$ by using the matrix relationship $\left(M^{I H}-\mu_{n} I\right) E^{n}=0$, for $n=1,4$, where $I$ is the identity matrix. Since these systems are homogenous, their solutions can be expressed as $\left(E_{x}^{1}, E_{z}^{1}, E_{h}^{1}\right)=E_{m}^{1}\left(e_{x}^{1}, e_{z}^{1}, e_{h}^{1}\right)$ and $\left(E_{x}^{4}, E_{m}^{4}, E_{h}^{4}\right)=$ $E_{z}^{4}\left(e_{x}^{4}, e_{m}^{4}, e_{h}^{4}\right)$, where $E_{m}^{1}$ and $E_{z}^{4}$ are arbitrary constants. Moreover, since $\mu_{4} \neq \frac{\partial m_{t+1}}{\partial m_{t}}$, it follows that $e_{m}^{4}=0$. Substituting all these equalities in the previous system, we get

$$
\begin{gathered}
e_{x}^{1}=-\frac{\frac{\partial x_{t+1}}{\partial h_{t}} \frac{\partial h_{t+1}}{\partial m_{t}}}{\frac{\partial x_{t+1}}{\partial h_{t}} \frac{\partial h_{t+1}}{\partial x_{t}}-\left(\frac{\partial h_{t+1}}{\partial h_{t}}-\mu_{1}\right)\left(\frac{\partial x_{t+1}}{\partial x_{t}}-\mu_{1}\right)}, \\
e_{z}^{1}=\frac{\frac{\partial z_{t+1}}{\partial x_{t}} \frac{\partial x_{t+1}}{\partial h_{t}} \frac{\partial h_{t+1}}{\partial m_{t}}-\left(\frac{\partial x_{t+1}}{\partial h_{t}} \frac{\partial h_{t+1}}{\partial x_{t}}-\left(\frac{\partial h_{t+1}}{\partial h_{t}}-\mu_{1}\right)\left(\frac{\partial x_{t+1}}{\partial x_{t}}-\mu_{1}\right)\right) \frac{\partial z_{t+1}}{\partial m_{t}}}{\left(\frac{\partial z_{t+1}}{\partial z_{t}}-\mu_{1}\right)\left(\frac{\partial x_{t+1}}{\partial h_{t}} \frac{\partial h_{t+1}}{\partial x_{t}}-\left(\frac{\partial h_{t+1}}{\partial h_{t}}-\mu_{1}\right)\left(\frac{\partial x_{t+1}}{\partial x_{t}}-\mu_{1}\right)\right)} \\
e_{h}^{1}=\frac{\frac{\partial h_{t+1}}{\partial m_{t}}\left(\frac{\partial x_{t+1}}{\partial x_{t}}-\mu_{1}\right)}{\frac{\partial x_{t+1}}{\partial h_{t}} \frac{\partial h_{t+1}}{\partial x_{t}}-\left(\frac{\partial h_{t+1}}{\partial h_{t}}-\mu_{1}\right)\left(\frac{\partial x_{t+1}}{\partial x_{t}}-\mu_{1}\right)} \\
e_{x}^{4}=-\frac{\frac{\partial z_{t+1}}{\partial z_{t}}-\mu_{4}}{\frac{\partial z_{t+1}}{\partial x_{t}}}
\end{gathered}
$$

and

$$
e_{h}^{4}=\frac{\frac{\partial h_{t+1}}{\partial x_{t}}\left(\frac{\partial z_{t+1}}{\partial z_{t}}-\mu_{4}\right)}{\frac{\partial z_{t+1}}{\partial x_{t}}\left(\frac{\partial h_{t+1}}{\partial h_{t}}-\mu_{4}\right)} .
$$

We must now establish the value of the arbitrary constants $E_{m}^{1}$ and $E_{z}^{4}$. First, $E_{m}^{4}=0$ implies that $m_{t}=E_{m}^{1} \mu_{1}^{t}+m$, so that $E_{m}^{1}=m_{0}-m$. Moreover, by construction,

$$
z_{t}=E_{z}^{1} \mu_{1}^{t}+E_{z}^{4} \mu_{4}^{t}+z=E_{m}^{1} e_{z}^{1} \mu_{1}^{t}+E_{z}^{4} \mu_{4}^{t}+z .
$$

This implies that

$$
E_{z}^{4}=\left(z_{0}-z\right)-\left(m_{0}-m\right) e_{z}^{1}
$$


Substituting the values of $E_{m}^{1}$ and $E_{z}^{4}$ we have obtained above into the equations characterizing the evolution of $x_{t}$ and $h_{t}$ around the BGP, we get

$$
\begin{aligned}
& x_{t}=\left(m_{0}-m\right) e_{x}^{1} \mu_{1}^{t}+\left[\left(z_{0}-z\right)-\left(m_{0}-m\right) e_{z}^{1}\right] e_{x}^{4} \mu_{4}^{t}+x, \\
& h_{t}=\left(m_{0}-m\right) e_{h}^{1} \mu_{1}^{t}+\left[\left(z_{0}-z\right)-\left(m_{0}-m\right) e_{z}^{1}\right] e_{h}^{4} \mu_{4}^{t}+h .
\end{aligned}
$$

Clearly, $x_{t}$ is non-monotonic around the steady state if there exists a positive finite number $t_{x}$ such that the derivative of the RHS of (44) with respect to $t$ equals zero at $t=t_{x}$. By differentiating (44) with respect to $t$, we obtain that the critical value of $t$ is given by

$$
t_{x}=\frac{\ln \left[-\left(\frac{\ln \left(\mu_{4}\right)}{\ln \left(\mu_{1}\right)}\right)\left(\frac{e_{x}^{4}}{e_{x}^{1}}\right)\left(\frac{z_{0}-z}{m_{0}-m}-e_{z}^{1}\right)\right]}{\ln \left(\frac{\mu_{1}}{\mu_{4}}\right)} .
$$

Similarly, it is obvious from (45) that $h_{t}$ is non-monotonic if

$$
t_{h}=\frac{\ln \left[-\left(\frac{\ln \left(\mu_{4}\right)}{\ln \left(\mu_{1}\right)}\right)\left(\frac{e_{h}^{4}}{e_{h}^{1}}\right)\left(\frac{z_{0}-z}{m_{0}-m}-e_{z}^{1}\right)\right]}{\ln \left(\frac{\mu_{1}}{\mu_{4}}\right)}
$$

is positive and finite. Consider the following configuration of parameter values:

$$
A=0.093, \sigma=5, \delta=0, n=0, \rho=0.03, \gamma=0.5, \text { and } \beta=0.2,
$$

and the initial values of the state variables are $z_{0}=0.99 z$ and $m_{0}=1.01 \mathrm{~m}$, so that (44) is a good approximation of the evolution of the growth rate $x_{t}$. Then, we obtain $t_{x}=5.9644$ and, hence, the growth rate $x_{t}$ displays a non-monotonic behavior around its stationary value. Consider now the same initial conditions for the state variables and the same set of parameter values as in the previous example, but making $A=0.183$ and $\delta=0.09$. In this case we get that $t_{h}=7.1723$ so that $h_{t}$ is non-monotonic around $h$.

Note that, if $B=0$ then $m_{t}=A$. In this case, $m_{0}=m$ and the equations (44) and (45) become

$$
\begin{aligned}
& x_{t}=\left(z_{0}-z\right) e_{x}^{4} \mu_{4}^{t}+x, \\
& h_{t}=\left(z_{0}-z\right) e_{h}^{4} \mu_{4}^{t}+h .
\end{aligned}
$$

In order to establish the relation between the variables $x_{t}$ and $h_{t}$ and $z_{0}$, we derive the sign of $e_{x}^{4}$ and $e_{h}^{4}$ in this case. First, note that $\frac{\partial z_{t+1}}{\partial z_{t}}=\mu_{2}>1>\mu_{4}$. Moreover,

$$
\begin{gathered}
\frac{\partial h_{t+1}}{\partial x_{t}}=-\frac{(1-\varepsilon h) \gamma}{\varepsilon h \varphi}<0, \\
\frac{\partial h_{t+1}}{\partial h_{t}}=\frac{\varepsilon h-\sigma}{(1-\sigma) \varepsilon h}=1+\frac{\sigma(1-\varepsilon h)}{(\sigma-1) \varepsilon h}>1>\mu_{4},
\end{gathered}
$$

and

$$
\frac{\partial z_{t+1}}{\partial x_{t}}=-\frac{(1+A-\delta) z}{(1+n) x^{2}}<0 .
$$

The previous inequalities imply that $e_{x}^{4}>0$ and $e_{h}^{4}>0$. Therefore, $x_{t}$ and $h_{t}$ increase (decrease) with time if $z_{0}<z\left(z_{0}>z\right)$ 
Proof of Proposition 6. Using (17), (19) and (27), we obtain the following Jacobian matrix evaluated at the BGP:

$$
M^{C J} \equiv\left(\begin{array}{ccc}
\frac{\partial x_{t+1}}{\partial x_{t}} & \frac{\partial x_{t+1}}{\partial z_{t}} & \frac{\partial x_{t+1}}{\partial m_{t}} \\
\frac{\partial z_{t+1}}{\partial x_{t}} & \frac{\partial z_{t+1}}{\partial z_{t}} & \frac{\partial z_{t+1}}{\partial m_{t}} \\
\frac{\partial m_{t+1}}{\partial x_{t}} & \frac{\partial m_{t+1}}{\partial z_{t}} & \frac{\partial m_{t+1}}{\partial m_{t}}
\end{array}\right)=\left(\begin{array}{ccc}
\frac{\gamma(\sigma-1)}{\sigma} & 0 & \frac{\beta x^{\beta}}{\sigma(A+1-\delta)} \\
-\frac{(1+A-\delta) z}{(1+n) x^{2}} & \frac{1+A-\delta}{(1+n) x} & \frac{z}{(1+n) x} \\
0 & 0 & x^{\beta-1}
\end{array}\right) .
$$

The eigenvalues of this matrix are: $\mu_{1}=x^{\beta-1}, \mu_{2}=\frac{1+A-\delta}{(1+n) x}$ and $\mu_{3}=\frac{\gamma(\sigma-1)}{\sigma}$. Note that $\mu_{1} \in(0,1)$ because $x>1$. Using the resource constraint (17), we see that $\mu_{2}=1+\frac{1}{(1+n) x}>1$. Finally, $\mu_{3} \in(0,1)$ because $\sigma \geq 1$. Therefore, the equilibrium path exhibits saddle path stability as the CJ model has two state variables, $z_{t}$ and $m_{t}$.

Proof of Proposition 7. Using Proposition 6, we obtain the following equation characterizing the equilibrium saddle path around the BGP for the CJ model:

$$
q_{t}=E^{1} \mu_{1}^{t}+E^{3} \mu_{3}^{t}+q
$$

where now $q_{t}=\left(x_{t}, m_{t}, z_{t}\right), \mu_{1}$ and $\mu_{3}$ are the stable eigenvalues, and $E^{1}=\left(E_{x}^{1}, E_{m}^{1}, E_{z}^{1}\right)$ and $E^{3}=\left(E_{x}^{3}, E_{m}^{3}, E_{z}^{3}\right)$ are the eigenvectors associated with the eigenvalues $\mu_{1}$ and $\mu_{3}$, respectively. We proceed to find some properties of the eigenvector $E^{1}$ and $E^{3}$ by using the matrix relationship $\left(M^{C J}-\mu_{n} I\right) E^{n}=0$, for $n=1,3$. Since these systems are homogenous, their solutions can be expressed as $\left(E_{x}^{1}, E_{z}^{1}\right)=E_{m}^{1}\left(e_{x}^{1}, e_{z}^{1}\right)$ and $\left(E_{x}^{3}, E_{m}^{3}\right)=E_{z}^{3}\left(e_{x}^{3}, e_{m}^{3}\right)$, where $E_{m}^{1}$ and $E_{z}^{3}$ are arbitrary constants. Moreover, since $\mu_{3} \neq \frac{\partial m_{t+1}}{\partial m_{t}}$, we get that $e_{m}^{3}=0$ and

$$
\begin{gathered}
e_{x}^{1}=-\frac{\frac{\partial x_{t+1}}{\partial m_{t}}}{\frac{\partial x_{t+1}}{\partial x_{t}}-\mu_{1}} \\
e_{z}^{1}=\frac{\frac{\partial z_{t+1}}{\partial x_{t}} \frac{\partial x_{t+1}}{\partial m_{t}}-\frac{\partial z_{t+1}}{\partial m_{t}}\left(\frac{\partial x_{t+1}}{\partial x_{t}}-\mu_{1}\right)}{\left(\frac{\partial z_{t+1}}{\partial z_{t}}-\mu_{1}\right)\left(\frac{\partial x_{t+1}}{\partial x_{t}}-\mu_{1}\right)},
\end{gathered}
$$

and

$$
e_{x}^{3}=-\frac{\frac{\partial z_{t+1}}{\partial z_{t}}-\mu_{3}}{\frac{\partial z_{t+1}}{\partial x_{t}}} .
$$

We must now establish the value of the arbitrary constants $E_{m}^{1}$ and $E_{z}^{3}$. First, $E_{m}^{3}=0$ implies that $m_{t}=E_{m}^{1} \mu_{1}^{t}+m$, so that $E_{m}^{1}=m_{0}-m$. Moreover, by construction,

$$
z_{t}=E_{z}^{1} \mu_{1}^{t}+E_{z}^{3} \mu_{3}^{t}+z=E_{m}^{1} e_{z}^{1} \mu_{1}^{t}+E_{z}^{3} \mu_{3}^{t}+z .
$$

This implies that

$$
E_{z}^{3}=\left(z_{0}-z\right)-\left(m_{0}-m\right) e_{z}^{1} .
$$

Substituting the values of $E_{m}^{1}$ and $E_{z}^{3}$ we have obtained above into the equation characterizing the evolution of $x_{t}$ around the BGP, we get

$$
x_{t}=\left(m_{0}-m\right) e_{x}^{1} \mu_{1}^{t}+\left[\left(z_{0}-z\right)-\left(m_{0}-m\right) e_{z}^{1}\right] e_{x}^{3} \mu_{3}^{t}+x .
$$


As in the proof of Proposition 5, we consider the parameter configuration (46) with the same initial conditions for the state variables. Then, we obtain that the critical value of $t$ that makes the derivative of the RHS of (47) equal to zero is $t_{x}=5.6603$ and, thus, $x_{t}$ turns out to be non-monotonic.

Note that, if $B=0$ then $m_{t}=A$. In this case, $m_{0}=m$ and the previous equation becomes

$$
x_{t}=\left(z_{0}-z\right) e_{x}^{3} \mu_{3}^{t}+x .
$$

In order to establish the relation between the variable $x_{t}$ and $z_{0}$, we derive the sign of $e_{x}^{3}$ in this case. First, note that $\frac{\partial z_{t+1}}{\partial z_{t}}>1>\mu_{3}$. Moreover,

$$
\frac{\partial z_{t+1}}{\partial x_{t}}=-\frac{(1+A-\delta) z}{(1+n) x^{2}}<0 .
$$

It then follows that $e_{x}^{3}>0$. Therefore, $x_{t}$ increases (decreases) with time if $z_{0}<z$ $\left(z_{0}>z\right)$. 